\title{
Social Network Modulation of Reward-Related Signals
}

\author{
Dominic S. Fareri, ${ }^{1 *}$ Michael A. Niznikiewicz, ${ }^{2 *}$ Victoria K. Lee, ${ }^{3}$ and Mauricio R. Delgado ${ }^{1}$ \\ ${ }^{1}$ Department of Psychology, Rutgers University, Newark, New Jersey 07102, ${ }^{2}$ Department of Psychology, University of Illinois at Urbana-Champaign, \\ Urbana, Illinois 61801, and ' ${ }^{3}$ epartment of Psychology and Neuroscience, Duke University, Durham, North Carolina 27708
}

Everyday goals and experiences are often shared with others who may hold different places within our social networks. We investigated whether the experience of sharing a reward differs with respect to social network. Twenty human participants played a card guessing game for shared monetary outcomes with three partners: a computer, a confederate (out of network), and a friend (in network). Participants subjectively rated the experience of sharing a reward more positively with their friends than the other partners. Neuroimaging results support participants' subjective reports, as ventral striatal BOLD responses were more robust when sharing monetary gains with a friend as compared to the confederate or computer, suggesting a higher value for sharing with an in-network partner. Interestingly, ratings of social closeness covaried with this activity, resulting in a significant partner $\times$ closeness interaction; exploratory analysis showed that only participants reporting higher levels of closeness demonstrated partner-related differences in striatal BOLD response. These results suggest that reward valuation in social contexts is sensitive to distinctions of social network, such that sharing positive experiences with in-network others may carry higher value.

\section{Introduction}

Social networks have seen increased public attention in recent years with the advent of social networking sites. Within one's social network, there may exist varying degrees of closeness between members (e.g., best friend, new acquaintance, business colleague), and, as such, we may place more value on experiences with close others as compared to those who are more distant or out of our social network. Often times we may pursue goals that will be shared with another (e.g., teaming up with a best friend to win a competition) and in doing so may take turns contributing toward these goals. An interesting question thus arises as to whether such shared experiences with a close, in-network other are experienced as more valuable than when working with someone out of network. Further, is the neural representation of shared rewards reflected in putative reward circuitry and also modulated by social network?

A rich literature highlighting the neural underpinnings of goal-directed behaviors and reward processing focuses on functional corticobasal ganglia loops (Middleton and Strick, 2000), with the striatum being a critical component (Robbins and Everitt, 1996; Delgado, 2007; Haber and Knutson, 2010). Blood oxygen level-dependent (BOLD) responses in the human striatum represent reward value during reward expectation or prediction (Knutson et al., 2001; O'Doherty et al., 2003a) as well as upon receiving positively and negatively valenced outcomes

\footnotetext{
Received February 8, 2012; revised April 6, 2012; accepted May 1, 2012.

Author contributions: D.S.F., M.A.N., and M.R.D. designed research; D.S.F., M.A.N., and V.K.L. performed research; D.S.F., M.A.N., V.K.L., and M.R.D. analyzed data; D.S.F. and M.R.D. wrote the paper.

This work was supported by National Institutes of Mental Health Grant R01MH084081 (M.R.D.). We thank Dr. Kent Harber for helpful discussion.

*D.S.F. and M.A.N. contributed equally to this work.

Correspondence should be addressed to Mauricio R. Delgado, Department of Psychology, Rutgers University, 101 Warren Street, Newark, NJ 07102. E-mail: delgado@psychology.rutgers.edu.

DOI:10.1523/JNEUROSCI.0610-12.2012

Copyright $\odot 2012$ the authors $\quad 0270-6474 / 12 / 329045-08 \$ 15.00 / 0$
}

(Delgado et al., 2000; Seymour et al., 2007) of differing magnitudes and incentives (Knutson et al., 2001; Galvan et al., 2005; Knutson et al., 2005). Recent efforts have investigated whether social reward valuation is similarly processed in the brain (Leotti and Delgado, 2011). Receiving another person's positive opinion of us, for example, elicits striatal activity similar to that seen when receiving monetary rewards (Izuma et al., 2010). Other types of social rewards, such as observing another person's success (Mobbs et al., 2009), and charitable giving (Harbaugh et al., 2007) also recruit the striatum, suggesting that social reward processing recruits neural circuitry similar to processing primary and secondary rewards. However, it remains unclear how reward valuation may be modulated as a function of social network.

To investigate this question, we administered a card guessing task for monetary outcomes (Delgado et al., 2000) in which we manipulated two experimental factors: participants' partners and roles (Fig. 1). Participants performed the task with three different partners with whom they shared monetary outcomes achieved during the task - a computer, a confederate, and, importantly, one of their close friends. Participants alternated roles with their partners during the task between being the player (e.g., making responses) and the observer (e.g., watching the other agents make responses). Using functional magnetic resonance imaging (fMRI), this design allowed us to probe whether the value of shared outcomes is modulated by both degree of social network and one's role (active/passive) in attaining them. We hypothesized that social network would modulate rewardrelated striatal signals; we expected greater BOLD responses when sharing positive outcomes with a close friend (in network) as compared to when sharing with a confederate (out of network) or a computer (nonsocial entity).

\section{Materials and Methods}

Participants. Twenty-three gender-matched participant pairs (12 female) took part in this experiment. Only one participant from each pair under- 
went fMRI (MRI participant); the other participant took part in the experiment from the control room in the University Heights Advanced Imaging Center at the University of Medicine and Dentistry of New Jersey (Newark, NJ). All MRI participants were screened for head trauma and history of psychiatric illness. Three participant pairs were excluded from further analysis, two due to excessive head motion by the MRI participant and one due to both reported discomfort with the scanner environment and reported lack of focus on task requirements. Data from 20 participants were included in the final analysis ( 10 females; mean age, 20.5 years; SD, 2.2 years). Analysis of subjective reports of closeness was also conducted in the cohort of participants who did not undergo fMRI (10 females; mean age, 20.6 years; SD, 2.2 years). All participants gave informed consent before participating in the experiment. The internal review boards of Rutgers University and the University of Medicine and Dentistry of New Jersey approved this study.

Experimental paradigm. The experiment took part over $2 \mathrm{~d}$. Day 1 involved completion of questionnaires probing social closeness, while the experimental session took place on day 2 . The focus of this study was to probe valuation of shared outcomes as a function of social network. Thus, our critical manipulation involves sharing outcomes with an innetwork (friend) versus an out-of-network (confederate) partner. Participants recruited from Rutgers University-Newark and the surrounding area were told to bring a close friend of the same gender (not a romantic partner or a family member) whom they felt they could count on. After giving informed consent on day 1, each member of the pair separately completed the Inclusion of Other in Self scale (IOS) (Aron et al., 1992), which consists of pairs of circles (one labeled self and one labeled other) varying in their degree of overlap. Greater overlap indicates greater relationship closeness. Participants were independently instructed to choose the pair of circles on the IOS that best characterized their relationship. We administered this measure motivated by the idea that there could be varying degrees of closeness with different members of one's social network. Thus, we deemed it important to measure how close MRI participants were with the friend they chose to bring with them. Both participants also completed a supporting measure, a modified version of the Personal Acquaintance Measure (PAM) (Starzyk et al., 2006), to assess relationship characteristics such as duration of acquaintance. A facial photograph was taken of the close friend to be programmed into the task as a stimulus as described below. Both participants were paid $\$ 10$ for their participation on day 1 and then instructed to return on the day of the scheduled fMRI experimental session (typical delay between sessions was 1 or $2 \mathrm{~d}$ ).

On day 2, the MRI participant was told that he or she would be playing a gambling game (Delgado et al., 2000) for monetary compensation (Fig. 1). We manipulated two factors here: MRI participants' (1) partners and (2) role during the task. MRI participants played the game with three different partners: a computer, a gender-matched confederate from the laboratory (whose photograph was already programmed into the task), and their close friend. Each confederate was portrayed as another participant, and his or her identity was not revealed until debriefing at the end of the fMRI session so participants would not suspect an unfair advantage in the task. The computer partner served as a nonsocial control, depicted by a photograph of a computer. MRI participants' roles also alternated between making choices in the game (player) and watching their partners choose (observer).

Participants guessed whether a card's value was higher $(6,7,8,9)$ or lower $(1,2,3,4)$ than the number 5 . The task consisted of 96 total trials, separated into 4 runs of 24 trials each. All runs consisted of 8 trials per partner condition, randomly presented. MRI participants' role (player/ observer) alternated between runs ( 2 player runs, 2 observer runs), the order of which was counterbalanced across participants. During player runs, MRI participants responded by pressing buttons designated "high" or "low" on a fiber optic response box (Current Designs), and the friend pressed a designated button on a keyboard to "release" the MRI participant's responses (i.e., allow them to be counted). During the observer runs, MRI participants made a similar button press to release their partners' responses. This served as a motor control and to ensure attentiveness in both MRI participants and their friends. Unbeknownst to MRI participants, confederate and computer responses were preprogrammed into the game for both role conditions. A correct guess (e.g., correctly guessing the card value was low) resulted in a monetary gain of $\$ 4$ (positive outcome), whereas an incorrect guess resulted in a monetary loss of \$2 (negative outcome). Failure to make or release responses (during player or observer runs, respectively) resulted in a missed trial and a monetary loss of $\$ 1$ to encourage responding. MRI participants were informed that all earned monetary outcomes would be shared equally between the participant and partner. Outcomes were predetermined (50\% reward, $50 \%$ punishment) to ensure an equivalent experience across participants and were presented randomly.

Each trial began with a response phase $(2 s)$ in which a question mark appeared within a card on a computer screen. Above the card was a picture of the MRI participant's partner (computer, confederate, or friend) for that particular trial, and below the card was a reminder of their role in that functional run (e.g., player/observer). Participants made the appropriate responses depending on their roles. This was followed by a jittered interstimulus interval $(4-10 s)$ and the outcome phase $(2 s)$. During the outcome phase, participants saw the same visual presentation as during the response phase, except that the question mark was replaced by the card's numerical value and either a green check mark (correct guess/monetary gain) or a red $\times$ (incorrect guess/monetary loss). Trials were separated by a jittered intertrial interval (12-14 s).

Following the experimental session, MRI participants subjectively rated how excited they were to win and how disappointed they were to lose with each partner using seven-point Likert scales $(1=$ not at all, $7=$ a lot). Additionally, they rated their partners on Likert scales of trustworthiness and liking.

The paradigm was programmed using E-Prime version 2.0 (PST). Participants viewed the stimuli in the fMRI scanner through a backprojection system. The close friend participated in the experiment using a standard keyboard and computer in the control room. MRI participants were guaranteed a base rate of $\$ 25$ per hour and were paid additionally based on the outcomes of the game. The friends were paid at a base rate of $\$ 10$ per hour and also received equal outcome-dependent payment for trials in which they were partnered with their friends, as earned outcomes between the partners were shared. Participants were 
fully debriefed as to the predetermined reinforcement schedule and the identity of the confederate at the end of the session. The use of deception in this task was necessary to maximize equivalence in the experience across participants with regard to the outcomes earned and the out-ofnetwork other (e.g., confederate's appearance, mannerisms). During debriefing, participants were informed of the reasons behind these two critical design aspects. One risk of the use of deception is that participants may not be as susceptible to the manipulation, particularly if they are suspicious of deception in experimental studies. However, this was not evident in post-experimental debriefing sessions.

Behavioral analysis. We examined whether MRI participants and friends viewed their relationships similarly with separate Pearson's correlations on IOS and PAM responses. Differences between MRI participants' IOS ratings of their friends and of the confederates were compared using paired samples $t$ tests; this was also done for friends' ratings of the MRI participants and of the confederates. Due to experimenter error, one MRI participant and two friends did not complete IOS ratings for the confederate. Post-session subjective ratings were analyzed with separate one-way repeated-measures ANOVAs in SPSS with partner as the factor. A Greenhouse-Geiser correction is reported when conditions for sphericity were violated. Reaction time data from the response phase was entered into a 2 (role) $\times 3$ (partner) repeated-measures ANOVA. All significant behavioral effects were further examined with post hoc $t$ tests. Wherever a family of post hoc tests consisted of two or more comparisons, we corrected for multiple comparisons by applying the sequential Bonferroni method (Holm, 1979; Rice, 1989). Missed trials were excluded from all analyses. No participants were excluded from analysis due to amount of missed trials, as none fell outside exclusionary criterion of $3 \mathrm{SD}$ from the group mean of missed trials (mean, 1.22; SD, 2.56).

Physiological assessment and analysis. Skin conductance responses (SCRs) were collected from the MRI participants using a BIOPAC Systems skin conductance module and analyzed using AcqKnowledge BIOPAC Systems software. Disposable electrodes were placed on the second and third fingers of each participant's left hand and connected to MRI-compatible electrode leads grounded through the BIOPAC Systems MRI filter system for biopotential amplifiers. SCRs were measured on every trial to the onset of the stimuli during the response phase and separately to the onset of stimuli during the outcome phase. The largest response that met the criterion of a minimum base-to-peak difference in SCRs of 0.02 microsiemens $(\mu \mathrm{S})$ between $0.5 \mathrm{~s}$ and $4.5 \mathrm{~s}$ after the onset of each stimulus was considered a valid response. Responses not meeting these criteria were scored as zero. Waveforms were preprocessed with a low pass filter (cutoff frequency of $25 \mathrm{~Hz}, 32$ coefficients) and mean value smoothing ( 3 samples). Analysis excluded missed trials, and SCRs were square root transformed before analysis (LaBar et al., 1995). Mean SCRs were entered into a 2 (role) $\times 3$ (partner) repeated-measures ANOVA during the response phase. To parallel analysis of neuroimaging data, we conducted 2 (role) $\times 3$ (partner) ANOVAs on wins and losses separately. Post hoc $t$ tests further examining resulting effects were corrected for multiple comparisons using the sequential Bonferroni method (Holm, 1979; Rice, 1989).

fMRI acquisition and analysis. Images were acquired using a 3T Siemens Allegra head-only scanner. Structural images were collected using a T1-weighted MPRAGE sequence $(256 \times 256$ matrix; FOV $=256 \mathrm{~mm}$; $1761 \mathrm{~mm}$ sagittal slices). Functional images were acquired using a singleshot gradient echo EPI sequence $(\mathrm{TR}=2000 \mathrm{~ms}$, TE $=25 \mathrm{~ms}$, FOV $=$ 192 , flip angle $=80^{\circ}$, bandwidth $=2604 \mathrm{~Hz} / \mathrm{Px}$, echo spacing $\left.=44\right)$ and comprised thirty-five contiguous oblique-axial slices $(3 \times 3 \times 3 \mathrm{~mm}$ voxels) parallel to the anterior commissure-posterior commissure line. BrainVoyager QX software (version 2.2; Brain Innovation) was used to preprocess and analyze the imaging data. Preprocessing consisted of 3D motion correction (six parameters), slice scan time correction (trilinear/ sinc interpolation), spatial smoothing, using a 3D Gaussian filter (4 mm FWHM), voxelwise linear detrending, and high-pass filtering of frequencies (3 cycles per time course). Structural and functional data of each participant were then transformed to standard Talairach stereotaxic space (Talairach and Tournoux, 1988). We created individual whole- brain (including gray and white matter) masks and combined them in an additive manner to create a group mask excluding the skull.

A random effects GLM was conducted using role (player/observer), partner (computer/confederate/friend), and outcome (positive/negative) as regressors. We modeled the response phase and the outcome phase separately such that the response phase included 6 regressors $(2$ levels of role, 3 levels of partner), and the outcome phase included 12 regressors (2 levels of role, 3 levels of partner, 2 levels of outcome). We also included 7 regressors of no interest ( 6 motion parameters, and missed trials). Regressors were convolved with a 2 -gamma hemodynamic response function and $z$-transformed at the single participant level. Statistical parametric maps (SPMs) were generated using the false discovery rate (FDR) method (Genovese et al., 2002). The outcome phase was thresholded at $q<0.01$ to ensure that observed robust BOLD activity was originating in the striatum. SPMs generated during the response phase were thresholded at $q<0.05$. Mean parameter estimates were extracted from clusters surviving FDR correction in both phases using 3D cluster spreads.

To investigate reward-related BOLD activity, we conducted a contrast of positive-negative outcomes based on previous research (Delgado et al., 2000; O’Doherty et al., 2003a) using a two-tailed $t$ test in BrainVoyager. This allowed us to additionally examine whether any regions demonstrated greater responses to losses compared to gains. Mean parameter estimates were extracted based on an average across all voxels in each ROI (centered around the peak) identified in this contrast and entered into two separate 2 (role) $\times 3$ (partner) repeated-measures ANOVAs: one for shared positive outcomes, and one for shared negative outcomes. We additionally entered MRI participants' responses on the IOS indicating closeness with their friends as a covariate into separate 2 (role) $\times 3$ (partner) repeated-measures ANCOVAs for shared positive and negative outcomes to probe the effects of social closeness. To visualize and explore an interaction between BOLD activation and participants' ratings of closeness with their friends, we split participants into high and low IOS groups based on their ratings of closeness with their friends: high IOS participants were those rating their friendship as above the midpoint on the scale; low IOS participants were those whose friendships were rated at or below the midpoint. This was a strictly arbitrary distinction made solely to visualize and understand the interaction.

The response phase, with three levels of partner, did not lend itself to analysis using a linear contrast. We thus conducted a 2 (role) $\times 3$ (partner) repeated-measures ANOVA in BrainVoyager. Mean parameter estimates were extracted from resulting ROIs exhibiting significant effects to characterize BOLD activity during the response phase. All significant effects in the outcome and response phases were examined with post hoc $t$ tests, and were corrected for multiple comparisons using the sequential Bonferroni method (Holm, 1979; Rice, 1989).

\section{Results}

\section{Behavioral results}

Relationship closeness was an important part of this study, as we were interested in investigating whether outcomes shared with an in-network compared to an out-of-network partner were experienced differently. To ensure that both participants in a pair had a similar view of their relationship closeness, we began by correlating MRI participant and friend responses on the IOS (Aron et al., 1992), which is a simple scale measuring social closeness. This revealed a strong positive correlation $(r=0.867, p<0.001)$. Correlating MRI participant and friend responses on a secondary measure, the Personal Acquaintance Measure (PAM) (Starzyk et al., 2006), which quantifies various dimensions of relationship strength (see Materials and Methods), supported the IOS results $(r=0.681, p<0.001)$, suggesting that both parties generally held a similar perception of their relationship. As a manipulation check, we additionally investigated how close both sets of participants felt to the confederate based on IOS responses. Not surprisingly, both MRI participants $\left(t_{(18)}=7.73, p<0.001\right)$ and 


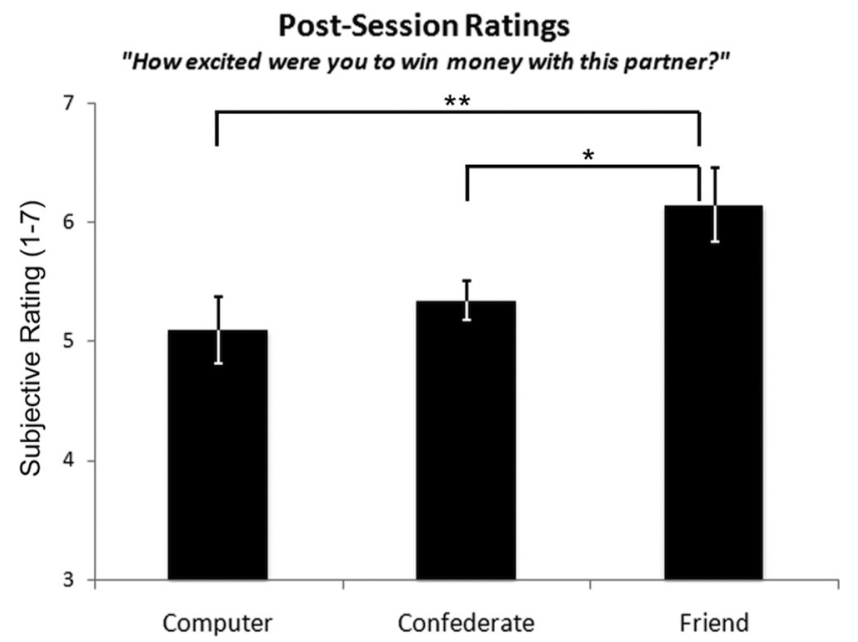

Figure 2. Post-session ratings. MRI participants completed subjective ratings of their experiences during the task. Ratings of excitement for winning with each partner demonstrated a main effect of partner: participants were significantly more excited to earn shared positive outcomes with their friend as compared to the confederate or computer ( \pm SEM; ${ }^{* *} p<0.016$, $\left.{ }^{*} p<0.025\right)$.

their friends $\left(t_{(17)}=7.40, p<0.001\right)$ reported feeling significantly closer to each other than to the confederate.

One-way repeated-measures ANOVAs probed MRI participants' subjective experiences with each partner post-session. A significant main effect was observed for ratings of excitement for shared positive outcomes $\left(F_{(1.52,27.79)}=6.36, p<0.01\right.$; Fig. 2$)$, where MRI participants were more excited to win with their friends as compared to the computer $\left(t_{(19)}=2.81, p=0.01\right)$ or the confederate $\left(t_{(19)}=2.56, p=0.02\right)$. No differences were observed in participants' excitement ratings for sharing positive outcomes with the confederate compared to the computer $\left(t_{(19)}=1.16, p=0.26\right)$, nor did we observe an effect for ratings of disappointment to shared negative outcomes across partners $\left(F_{(2,38)}=2.30, p=0.114\right)$. These post-session subjective ratings demonstrate that positive experiences with a close friend (in network) were generally viewed as more favorable than those with an out-of-network human (confederate) with whom there was no established relationship or a nonsocial entity (computer).

Potential reaction time differences during the response phase as a function of MRI participants' role and partner were probed. A $2 \times 3$ repeated-measures ANOVA revealed a significant main effect of role $\left(F_{(1,19)}=8.514, p<0.01\right)$, such that MRI participants exhibited longer reaction times as the players than as the observers $\left(t_{(19)}=2.92, p<0.01\right)$. This analysis did not reveal a significant main effect of partner $\left(F_{(2,38)}=1.057, p>0.3\right)$ or a significant role $\times$ partner interaction $\left(F_{(2,38)}=0.599, p>0.5\right)$.

\section{Physiological results}

SCRs were acquired to examine whether MRI participants' physiological arousal differed as a function of role and partner during the response phase. Results from a $2 \times 3$ repeated-measures ANOVA revealed a significant main effect of partner $\left(F_{(2,38)}=\right.$ $4.65, p<0.05)$. Post hoc $t$ tests demonstrated that this main effect was driven by trials on which MRI participants were partnered with their friends; average SCRs during the response phase of friend trials were significantly higher than during computer $\left.t_{(19)}=3.20, p=0.005\right)$ trials and marginally significantly greater (after correcting for multiple comparisons using the sequential Bonferroni method) than on confederate trials $\left(t_{(19)}=2.33, p=\right.$
Table 1. Regions activated in a contrast of all positive $>$ all negative outcomes

\begin{tabular}{|c|c|c|c|c|c|c|c|}
\hline \multirow[b]{2}{*}{ Region of activation } & \multirow[b]{2}{*}{ BA } & \multirow[b]{2}{*}{ Laterality } & \multicolumn{3}{|c|}{$\begin{array}{l}\text { Talairach } \\
\text { coordinates }\end{array}$} & \multirow[b]{2}{*}{ Voxels $\left(\mathrm{mm}^{3}\right)$} & \multirow[b]{2}{*}{ Fstatistic } \\
\hline & & & $x$ & $y$ & $Z$ & & \\
\hline Caudate nucleus & & Right & 17 & 10 & 18 & 42 & 6.95 \\
\hline Medial frontal gyrus & 10 & Right & 2 & 52 & 12 & 195 & 6.48 \\
\hline Putamen & & Right & 26 & -5 & 6 & 169 & 7.38 \\
\hline Medial temporal lobe & 30 & Right & 20 & -38 & 0 & 49 & 6.94 \\
\hline Ventral striatum & & Left & -13 & 7 & 0 & 2740 & 9.79 \\
\hline Ventral striatum & & Right & 11 & 1 & -6 & 2713 & 8.77 \\
\hline
\end{tabular}

Win $>$ loss, FDR corrected; $q<0.01$

0.03). Two separate $2 \times 3$ repeated-measures ANOVAs at the time of outcome were also conducted on SCRs to shared wins and losses, respectively. No significant effects were observed for either wins or losses.

\section{Neuroimaging results}

Outcome phase: win trials

Our question of interest was whether BOLD responses associated with shared monetary outcomes were modulated by degree of social network, particularly in the striatum. We conducted a contrast of all positive (wins)-all negative (losses) outcomes to identify regions sensitive to positive outcomes, correcting for multiple comparisons at a FDR $q<0.01$. Mean parameter estimates from regions identified by this contrast were then entered into two separate $2 \times 3$ repeated-measures ANOVAs, one for positive and one for negative outcomes.

Contrasting positive versus negative shared outcomes yielded activation in several regions (Table 1 ) but was particularly robust bilaterally in the ventral striatum (Fig. $3 A$ ) as in previous studies (Delgado et al., 2000). A $2 \times 3$ repeated-measures ANOVA investigating effects of role and partner during trials resulting in positive outcomes (wins) in the right ventral striatum $(x, y, z=$ $11,1,-6)$ revealed a significant main effect of partner $\left(F_{(2,38)}=\right.$ 5.244, $p<0.02)$. Positive outcomes shared with one's friend elicited significantly greater BOLD responses in the right ventral striatum as compared to those shared with the computer $\left(t_{(19)}=\right.$ $2.71, p=0.014)$ or the confederate $\left(t_{(19)}=2.49, p=0.022\right.$; Fig. $3 B)$. A significant main effect of role was also observed $\left(F_{(1,19)}=\right.$ 5.897, $p<0.03$ ) such that BOLD responses to shared positive outcomes in the right ventral striatum were significantly greater during the player condition as compared to the observer condition $\left(t_{(19)}=2.43, p<0.03\right)$. Conducting this same ANOVA on extracted mean parameter estimates in the left ventral striatum $(x, y, z=-13,7,0)$ also revealed a main effect of partner $\left(F_{(1.55,29.40)}=5.73, p<0.02\right)$, such that BOLD activity to winning with one's friend was significantly higher than when winning with the computer $\left(t_{(19)}=2.59, p=0.018\right)$ or the confederate $\left(t_{(19)}=2.67, p=0.015\right)$. A significant main effect of role was additionally observed in this region $\left(F_{(1,19)}=5.18, p<0.04\right)$ showing greater responses during the player compared to the observer condition $\left(t_{(19)}=2.26, p<0.04\right)$. No interaction effects were observed in either the left $\left(F_{(2,38)}=1.06, p>0.3\right)$ or the right $\left(F_{(2,38)}=0.59, p>0.5\right)$ ventral striatum.

Because a motivating factor for this study was investigating the effect of sharing a positive outcome with a close friend compared to an unknown other, we conducted a role $\times$ partner ANCOVA entering MRI participants' ratings of their friends on the IOS as a covariate. Interestingly, this analysis revealed a significant partner $\times$ IOS interaction in the right ventral striatum $\left(F_{(2,36)}=3.42, p<0.05\right)$. To visualize and explore this interaction, we split MRI participants based on their ratings of their 


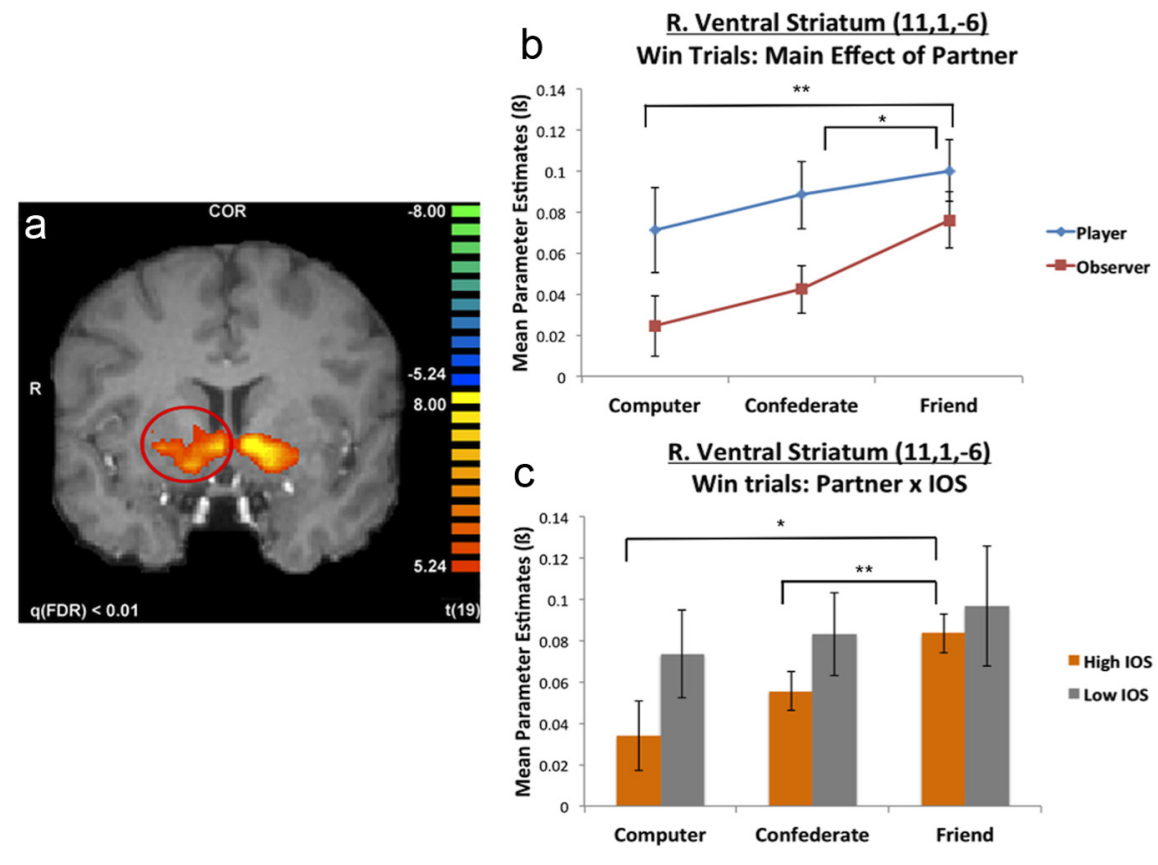

Figure 3. Neuroimaging results: outcome phase. $\boldsymbol{a}$, A contrast of positive $>$ negative outcomes revealed bilateral ventral striatal activity $(q(\mathrm{FDR})<0.01)$. $\boldsymbol{b}, \mathrm{A} 2$ (role) $\times 3$ (partner) repeated-measures ANOVA on extracted mean parameter estimates from right ventral striatum in response to positive outcomes demonstrated a significant main effect of partner, showing strongest BOLD activation when sharing positive outcomes with a friend $\left( \pm S E M ;{ }^{* *} p<0.016,{ }^{*} p<0.025\right)$. (c). Including MRI participants IOS ratings of their friends as a covariate with right ventral striatal BOLD activation to positive outcomes revealed a significant partner $\times 10$ S interaction: only high closeness $(n=13$; orange bars) participants show significant differences in right ventral striatal BOLD activation as a function of partner $\left( \pm\right.$ SEM; $\left.{ }^{* *} p<0.016,{ }^{*} p<0.05\right)$. Low closeness participants $(n=7$; gray bars) do not exhibit these differences, though they tend to show increased BOLD responses regardless of partner.

friends on the IOS. We classified participants who rated their relationship at the midpoint or lower on the IOS scale as low IOS (7 participants) and those rating their relationship above the midpoint as high IOS (13 participants). This exploratory analysis suggested that in high IOS participants, striatal BOLD responses to sharing positive outcomes with a friend were greater than responses to sharing with the computer $\left(t_{(12)}=2.51, p=0.028\right.$; trend when correcting for multiple comparisons) or confederate $\left(t_{(12)}=3.89, p=0.002\right)$. No differences were observed between responses to outcomes shared with the computer and confederate $\left(t_{(12)}=1.28, p=0.22\right)$. Low IOS participants demonstrated no significant partner-related differences in striatal BOLD responses (Fig. 3C).

A significant cluster of activity in medial prefrontal cortex, specifically in Brodmann area (BA) $10(x, y, z=2,52,12)$ also emerged when contrasting positive-negative outcomes. A $2 \times 3$ repeated-measures ANOVA on mean parameter estimates demonstrated a significant main effect of partner during shared positive outcomes $\left(F_{(2,38)}=4.03, p<0.03\right)$ driven by BOLD responses with the computer being significantly lower than those with the confederate $\left(t_{(19)}=3.14, p=0.005\right)$ and friend $\left(t_{(19)}=\right.$ $2.54, p=0.02)$. Unlike the striatum, no differences were observed in the BOLD response to shared positive outcomes here between friend and confederate. A main effect of role or interaction effect was not observed in this region. Additionally, including IOS ratings of closeness with one's friend as a covariate did not reveal any significant effects in this region.

Outcome phase: loss trials

As the contrast of all positive-all negative outcomes was a twotailed $t$ test, it would also have identified any regions more sensitive to losses; however, no significant clusters emerged showing this pattern. To probe losses further, we conducted a $2 \times 3$ repeated-measures ANOVA on mean parameter estimates extracted from the right ventral striatum ROI on trials resulting in a negative outcome (losses) only. This analysis revealed only a main effect of role in the right ventral striatum $\left(F_{(1,19)}=4.529, p<0.05\right)$. This was characterized by greater activity when participants were in the observer role compared to the player role $\left(t_{(19)}=\right.$ $2.13, p<0.05)$. This effect was not observed in the left ventral striatum, nor was there a main effect of partner or an interaction effect in either the left or the right ventral striatum during shared losses.

Response phase: main effect of partner

The response phase was investigated with a $2 \times 3$ whole-brain repeated-measures ANOVA using role and partner as withinsubjects factors, respectively, correcting for multiple comparisons using a FDR of $q<0.05$. Regions identified by this analysis showing a significant main effect of partner are noted in Table 2. Within this set of regions, a cluster in the right ventral caudate nucleus $(x, y, z=8,7,3)$ demonstrated increased BOLD activation during the response phase of friend trials compared to computer $\left(t_{(19)}=4.55, p=\right.$ $0.0002)$ or confederate $\left(t_{(19)}=2.71, p=\right.$ $0.0142)$ trials. Activity during confederate trials was also greater than during computer trials $\left(t_{(19)}=2.36, p=0.03\right)$. A large cluster in the medial prefrontal cortex encompassing portions of BA9, BA10, and BA32 also emerged in this analysis (Fig. 4A). Based on reported functional heterogeneity of these subregions of mPFC (Amodio and Frith, 2006) and on the presence of multiple peak voxels in this cluster, mean parameter estimates were initially extracted using $10^{3}$ voxel spreads around the different peaks (Table 2). However, as all of these subregions demonstrated the same pattern of results, we report results from the peak of the entire mPFC cluster $(x, y, z=-10,40,12)$. Consistent with the pattern of activity in the right ventral caudate nucleus, this cluster demonstrated increased BOLD activity during the response phase of friend trials as compared to computer $\left(t_{(19)}=\right.$ $6.43, p=0.000004)$ or confederate $\left(t_{(19)}=2.75, p=0.013\right)$ trials (Fig. $4 B$ ). BOLD activity in this $\mathrm{mPFC}$ cluster was also greater during the response phase of confederate as compared to computer trials $\left(t_{(19)}=4.76, p=0.0001\right)$.

We also investigated whether any regions demonstrated a significant main effect of role or an interaction between role and partner during the response phase. No voxels survived correction for multiple comparisons at a FDR $q<0.05$ when considering either the main effect or interaction.

\section{Discussion}

The current experiment investigated whether the experience and valuation of rewards, characterized primarily by striatal BOLD responses, are modulated by the social context in which they are attained. Our results suggest that sharing a positive outcome with an in-network other elicits greater subjective feelings of excitement and enhanced striatal reward value signals than sharing 
Table 2. Regions showing a main effect of partner in a 2 (role) $\times 3$ (partner) whole-brain ANOVA

\begin{tabular}{|c|c|c|c|c|c|c|c|}
\hline \multirow[b]{2}{*}{ Region of activation } & \multirow[b]{2}{*}{ BA } & \multirow[b]{2}{*}{ Laterality } & \multicolumn{3}{|c|}{ Talairach coordinates } & \multirow[b]{2}{*}{ Voxels $\left(\mathrm{mm}^{3}\right)$} & \multirow[b]{2}{*}{ Fstatistic } \\
\hline & & & $x$ & $y$ & $z$ & & \\
\hline Medial frontal gyrus & 9 & Left & -10 & 37 & 33 & 661 & 17.37 \\
\hline Precuneus & $31 / 18$ & Left & -4 & -62 & 27 & 685 & 13.36 \\
\hline Superior temporal gyrus & 22 & Right & 53 & -44 & 21 & 34 & 13.01 \\
\hline Middle temporal gyrus & 39 & Right & 41 & -65 & 18 & 180 & 15.37 \\
\hline Middle temporal gyrus & 39 & Left & -46 & -62 & 18 & 1094 & 28.02 \\
\hline Middle temporal gyrus & 39 & Right & 35 & -53 & 12 & 160 & 14.22 \\
\hline Putamen & & Left & -16 & 10 & 6 & 96 & 15.93 \\
\hline Ventral caudate nucleus & & Right & 8 & 7 & 3 & 28 & 14.03 \\
\hline Middle temporal gyrus & 21 & Right & 47 & -23 & -3 & 102 & 18.34 \\
\hline Basal forebrain & & Left & -1 & 1 & -3 & 578 & 19.23 \\
\hline Medial frontal gyrus & 10 & Right & 2 & 58 & -3 & 44 & 16.74 \\
\hline Middle temporal gyrus & 21 & Left & -58 & -5 & -12 & 147 & 12.88 \\
\hline Fusiform gyrus & 37 & Right & 35 & -53 & -18 & 561 & 22.73 \\
\hline Inferior frontal gyrus & 47 & Right & 29 & 22 & -21 & 119 & 13.92 \\
\hline Fusiform gyrus & 37 & Left & -43 & -44 & -24 & 56 & 12.30 \\
\hline
\end{tabular}

Response-phase ANOVA: main effect of partner, FDR corrected; $q<0.05$.

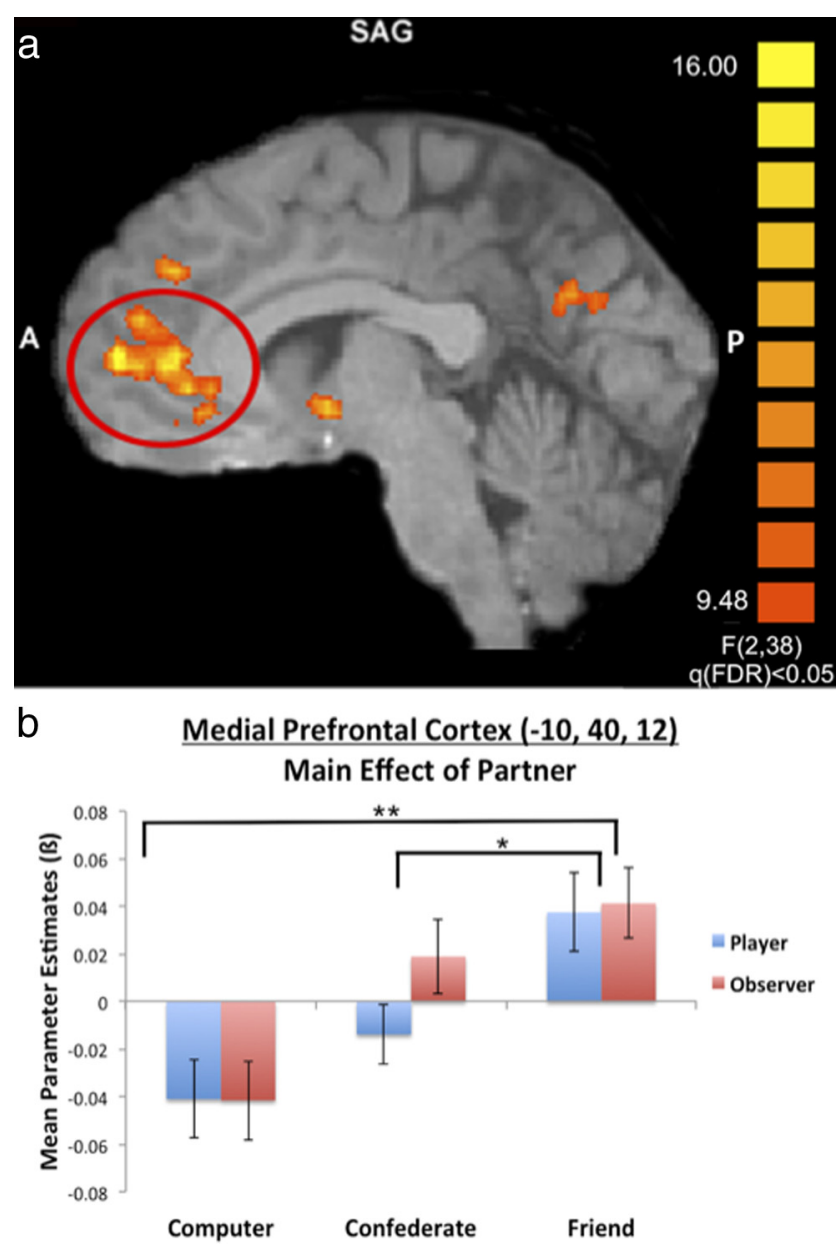

Figure 4. Neuroimaging results: response phase. $a, A 2$ (role) $\times 3$ (partner) ANOVA during the response phase revealed a main effect of partner $(q(\mathrm{FDR})<0.05)$ in medial prefrontal cortex. A, Anterior; $P$, posterior. $\boldsymbol{b}$, Extracted mean parameter estimates reveal that this effect was driven by significantly increased activity during the response phase of friend trials, as compared to confederate or computer trials ( \pm SEM; ${ }^{* *} p<0.016,{ }^{*} p<0.025$ ). with an out of network other or a nonsocial entity. This pattern of activity significantly covaried with a subjective measure of social closeness with a friend, suggesting that closeness may be a potential mechanism underlying the in-network versus out-ofnetwork difference observed in the striatum. Exploratory analysis of this interaction suggested that MRI participants reporting high levels of closeness with their friend demonstrated greater striatal BOLD responses to shared outcomes with their friend compared to other partners, whereas MRI participants reporting low closeness did not show this pattern. Interestingly, the modulatory influence of social network during receipt of shared positive outcomes did not extend to activity in more putatively known social regions such as the medial prefrontal cortex (Amodio and Frith, 2006), nor did we observe significant effects of partner during shared losses. Thus, the results suggest that sharing positive outcomes with a close, in-network other may carry higher value than sharing the same outcome with an unknown other in this experimental paradigm.

Emerging evidence supports an overlap in neural mechanisms processing motivated behaviors during social and nonsocial contexts (Rilling and Sanfey, 2011). Voluntary charitable giving, for example, elicits increases in ventral striatal BOLD activity (Harbaugh et al., 2007) in a manner akin to personal monetary gain (Izuma et al., 2010), and learning from social rewards (Jones et al., 2011; Lin et al., 2011) engages the striatum similarly to when learning in nonsocial contexts. Our study extends these findings, suggesting that the representation of reward value as reflected in striatal BOLD activation is sensitive to distinctions of social network. This interpretation is consistent with reports of nonsocial contextual modulation of experienced reward value signals in the ventral striatum (De Martino et al., 2009). In the current experiment, value signals differed with respect to social context within which rewarding outcomes were experienced (with an innetwork vs out-of-network partner).

One mechanism potentially underlying the social network modulation of value computation in the striatum is the degree of closeness with the in-network other. We observed an interaction between ratings of closeness with an in-network other and striatal 
BOLD responses during shared positive outcomes. Exploratory analysis suggested that the in-/out-network distinction in the striatum may be more prevalent in participants reporting higher levels of closeness. Social closeness involves merging representations of self and other (Aron et al., 1992). Perhaps with higher levels of closeness, achieving a shared reward may additionally serve to reaffirm the relationship. Though this is speculative, closeness in this cohort of participants may be a potential mechanism contributing to the computing value of shared rewards. Future studies targeting closeness may probe it by including different members of the same social network with varying degrees of closeness.

Importantly, our design allowed participants to experience shared outcomes regardless of whether they were directly responsible for them. We believe this is akin to experiences encountered in everyday life (e.g., taking turns contributing to a joint project). Increases in striatal BOLD responses are commonly associated with processing of primary (O'Doherty et al., 2003b) and monetary (Delgado et al., 2000) rewards for the self. Because a "self" condition was not included in our design, we cannot compare how rewards are valued when shared or not shared. Given previous reports of striatum activation when earning money for oneself as when playing for a charity (Harbaugh et al., 2007) or receiving social approval (Izuma et al., 2010), we can speculate that working toward a shared reward, particularly with someone within one's social network, recruits similar striatal regions as when playing for oneself-perhaps even more robustly.

The current study is unique in that all positive outcomes were shared between the MRI participants and their partners, and an enhancement in striatal reward value signals is observed when the partner is in-network. However, mechanisms other than closeness may be contributing to our results. Increased social abilities in high closeness participants might have facilitated better differentiation of partners (social/nonsocial and in network/out of network). Our exploratory analysis of high and low IOS participants provides some evidence against this, as high IOS participants demonstrated no difference in striatal BOLD response to outcomes shared with the computer and those shared with the confederate. If high IOS participants were more social in general, we might have observed a significant difference between these two conditions (i.e., confederate $>$ computer).

It is also possible that some component of the increased subjective and neural responses to sharing positive outcomes with an in-network partner was driven by MRI participants' empathic abilities. Empathy facilitates the development of close relationships (van Winden et al., 2008) by allowing emotional responses toward another person and perspective taking (Lamm et al., 2007). Studies of empathy have typically focused on the negative domain (e.g., pain), reporting circuitry (e.g., insula, dorsal ACC) (Singer et al., 2004) not observed in our study during positive outcomes. It is certainly plausible that differences in empathic ability may be an alternative or additional contributor to increased BOLD responses when sharing outcomes with an innetwork other.

Another consideration is that ventral striatal BOLD activity often correlates with reward prediction error signals during affective learning paradigms (O'Doherty et al., 2003b; O'Doherty et al., 2004; Schonberg et al., 2007; Glascher et al., 2010). The signal observed in this task may encompass some properties of a prediction error learning signal: participants might have had different expectations based on the partner with whom they were interacting. Due to the task structure and the predetermined nature of outcome presentation, participants did not have a true opportu- nity to learn a "correct" behavioral strategy in pursuit of more positive outcomes. Nevertheless, positive prediction errors might have been experienced during positive outcomes and further enhanced when sharing with a close, in-network other.

Social network did not exert an influence during shared negative outcomes, potentially due to the shared, cooperative context of the task and its focus on positive outcomes. While evidence supports striatal involvement when deciding to cooperate for positive outcomes (Rilling et al., 2002; King-Casas et al., 2005), the striatum may be sensitive to negative outcomes in more competitive contexts, such as losing a blind auction to an opponent (Delgado et al., 2008) or learning from a competitor's loss (Howard-Jones et al., 2010). The shared nature of negative outcomes in the present study might have decreased their salience, partially explaining the lack of partner-related differences in the loss domain. Future studies may probe whether lossrelated BOLD activity during a competition might also vary with respect to social network.

A main effect of partner was also observed in mPFC (BA10, bordering the anterior cingulate), during receipt of shared positive outcomes, driven by a social (friend/confederate) versus a nonsocial (computer) difference. This is consistent with findings implicating $\mathrm{mPFC}$ when cooperating with another person for a reward compared to playing alone (Elliott et al., 2006). Ventral mPFC regions differentiate monetary outcomes of varying magnitude (Knutson et al., 2003), and portions of the anterior cingulate track action outcome history, facilitating learning of optimal behavior in both nonsocial (Behrens et al., 2007) and social (Behrens et al., 2008) situations. The mPFC activity observed during the outcome phase in the present study may also represent tracking of the experienced outcome's social value, as functional dissociations have been observed in anterior cingulate when processing social outcomes and expectation violations (Somerville et al., 2006; Behrens et al., 2008). Our results provide further evidence implicating $\mathrm{mPFC}$ in processing socially relevant information and tracking social value.

A main effect of partner in BA9, BA10, and BA32 of mPFC during the response phase was characterized by a social network effect. Evidence implicates $\mathrm{mPFC}$ in various social processes (Kelley et al., 2002; Ochsner et al., 2004; Amodio and Frith, 2006) relating to self and close or similar others (Heatherton et al., 2006; Mitchell et al., 2006; Krienen et al., 2010). Electrophysiological recordings in the cingulate gyrus of nonhuman primates corroborate these results, showing its importance in detecting socially valued others (e.g., dominant members) (Rudebeck et al., 2006). The response phase mPFC activation in our task may similarly reflect the detection of socially valued others. This is supported by SCR results, which, although not necessarily sensitive enough to track outcome value as a function of partner, suggest highest arousal during the response phase of friend trials.

We often seek out positive experiences in our lives, and many times we will work with others to achieve them. In this study, we investigated whether the level of social network of a partner would affect the valuation of an achieved shared outcome. Our results suggest that, particularly when sharing positive experiences with another, the relationship with one's partner modulates its subjective experience and neural representation, supporting the interpretation that processing of shared positive outcomes is susceptible to social influence.

\section{References}

Amodio DM, Frith CD (2006) Meeting of minds: the medial frontal cortex and social cognition. Nat Rev Neurosci 7:268-277. 
Aron A, Aron EN, Smollan D (1992) Inclusion of the other in the self scale and the structure of interpersonal closeness. J Pers Soc Psychol 63:596-612.

Behrens TE, Woolrich MW, Walton ME, Rushworth MF (2007) Learning the value of information in an uncertain world. Nat Neurosci 10:1214-1221.

Behrens TE, Hunt LT, Woolrich MW, Rushworth MF (2008) Associative learning of social value. Nature 456:245-249.

Delgado MR (2007) Reward-related responses in the human striatum. Ann N Y Acad Sci 1104:70-88.

Delgado MR, Nystrom LE, Fissell C, Noll DC, Fiez JA (2000) Tracking the hemodynamic responses to reward and punishment in the striatum. J Neurophys 84:3072-3077.

Delgado MR, Schotter A, Ozbay EY, Phelps EA (2008) Understanding overbidding: using the neural circuitry of reward to design economic auctions. Science 321:1849-1852.

De Martino B, Kumaran D, Holt B, Dolan RJ (2009) The neurobiology of reference-dependent value computation. J Neurosci 29:3833-3842.

Elliott R, Völlm B, Drury A, McKie S, Richardson P, Deakin JF (2006) Cooperation with another player in a financially rewarded guessing game activates regions implicated in theory of mind. Soc Neurosci 1:385-395.

Galvan A, Hare TA, Davidson M, Spicer J, Glover G, Casey BJ (2005) The role of ventral frontostriatal circuitry in reward-based learning in humans. J Neurosci 25:8650-8656.

Genovese CR, Lazar NA, Nichols T (2002) Thresholding of statistical maps in functional neuroimaging using the false discovery rate. Neuroimage 15:870-878.

Gläscher J, Daw N, Dayan P, O'Doherty JP (2010) States versus rewards: dissociable neural prediction error signals underlying model-based and model-free reinforcement learning. Neuron 66:585-595.

Haber SN, Knutson B (2010) The reward circuit: linking primate anatomy and human imaging. Neuropsychopharmacology 35:4-26.

Harbaugh WT, Mayr U, Burghart DR (2007) Neural responses to taxation and voluntary giving reveal motives for charitable donations. Science 316:1622-1625.

Heatherton TF, Wyland CL, Macrae CN, Demos KE, Denny BT, Kelley WM (2006) Medial prefrontal activity differentiates self from close others. Soc Cogn Affect Neurosci 1:18-25.

Holm S (1979) A simple sequentially rejective multiple test procedure. Scand J Stat 6:65-70.

Howard-Jones PA, Bogacz R, Yoo JH, Leonards U, Demetriou S (2010) The neural mechanisms of learning from competitors. Neuroimage 53:790799.

Izuma K, Saito DN, Sadato N (2010) Processing of the incentive for social approval in the ventral striatum during charitable donation. J Cogn Neurosci 22:621-631.

Jones RM, Somerville LH, Li J, Ruberry EJ, Libby V, Glover G, Voss HU, Ballon DJ, Casey BJ (2011) Behavioral and neural properties of social reinforcement learning. J Neurosci 31:13039-13045.

Kelley WM, Macrae CN, Wyland CL, Caglar S, Inati S, Heatherton TF (2002) Finding the self? An event-related fMRI study. J Cogn Neurosci 14:785-794.

King-Casas B, Tomlin D, Anen C, Camerer CF, Quartz SR, Montague PR (2005) Getting to know you: reputation and trust in a two-person economic exchange. Science 308:78-83.

Knutson B, Adams CM, Fong GW, Hommer D (2001) Anticipation of increasing monetary reward selectively recruits nucleus accumbens. J Neurosci 21:RC159.

Knutson B, Fong GW, Bennett SM, Adams CM, Hommer D (2003) A region of mesial prefrontal cortex tracks monetarily rewarding outcomes: characterization with rapid event-related fMRI. Neuroimage 18:263-272.

Knutson B, Taylor J, Kaufman M, Peterson R, Glover G (2005) Distributed neural representation of expected value. J Neurosci 25:4806-4812.

Krienen FM, Tu PC, Buckner RL (2010) Clan mentality: evidence that the medial prefrontal cortex responds to close others. J Neurosci 30:13906-13915.
LaBar KS, LeDoux JE, Spencer DD, Phelps EA (1995) Impaired fear conditioning following unilateral temporal lobectomy in humans. J Neurosci 15:6846-6855.

Lamm C, Batson CD, Decety J (2007) The neural substrate of human empathy: effects of perspective-taking and cognitive appraisal. J Cogn Neurosci 19:42-58.

Leotti LA, Delgado MR (2011) Processing social and nonsocial rewards in the human brain. In: The oxford handbook of social neuroscience (Decety J, Cacioppo JT, eds), pp 178-194. New York: Oxford UP.

Lin A, Adolphs R, Rangel A (2012) Social and monetary reward learning engage overlapping neural substrates. Soc Cogn Affect Neurosci $7: 274-281$

Middleton FA, Strick PL (2000) Basal ganglia and cerebellar loops: motor and cognitive circuits. Brain Res Rev 31:236-250.

Mitchell JP, Macrae CN, Banaji MR (2006) Dissociable medial prefrontal contributions to judgments of similar and dissimilar others. Neuron 50:655-663.

Mobbs D, Yu R, Meyer M, Passamonti L, Seymour B, Calder AJ, Schweizer S, Frith CD, Dalgleish T (2009) A key role for similarity in vicarious reward. Science 324:900.

Ochsner KN, Knierim K, Ludlow DH, Hanelin J, Ramachandran T, Glover G, Mackey SC (2004) Reflecting upon feelings: an fMRI study of neural systems supporting the attribution of emotion to self and other. J Cogn Neurosci 16:1746-1772.

O'Doherty JP, Dayan P, Friston K, Critchley H, Dolan RJ (2003b) Temporal difference models and reward-related learning in the human brain. Neuron 38:329-337.

O’Doherty J, Critchley H, Deichmann R, Dolan RJ (2003a) Dissociating valence of outcome from behavioral control in human orbital and ventral prefrontal cortices. J Neurosci 23:7931-7939.

O’Doherty J, Dayan P, Schultz J, Deichmann R, Friston K, Dolan RJ (2004) Dissociable roles of ventral and dorsal striatum in instrumental conditioning. Science 304:452-454.

Rice WR (1989) Analyzing tables of statistical tests. Evolution 6:223-225.

Rilling JK, Sanfey AG (2011) The neuroscience of social decision-making. Annu Rev Psych 62:23-48.

Rilling J, Gutman D, Zeh T, Pagnoni G, Berns G, Kilts C (2002) A neural basis for social cooperation. Neuron 35:395-405.

Robbins TW, Everitt BJ (1996) Neurobehavioural mechanisms of reward and motivation. Curr Opin Neurobiol 6:228-236.

Rudebeck PH, Buckley MJ, Walton ME, Rushworth MF (2006) A role for the macaque anterior cingulate gyrus in social valuation. Science 313:1310-1312.

Schönberg T, Daw ND, Joel D, O’Doherty JP (2007) Reinforcement learning signals in the human striatum distinguish learners from nonlearners during reward-based decision making. J Neurosci 27:12860-12867.

Seymour B, Daw N, Dayan P, Singer T, Dolan R (2007) Differential encoding of losses and gains in the human striatum. J Neurosci 27:4826-4831.

Singer T, Seymour B, O’Doherty J, Kaube H, Dolan RJ, Frith CD (2004) Empathy for pain involves the affective but not sensory components of pain. Science 303:1157-1162.

Somerville LH, Heatherton TF, Kelley WM (2006) Anterior cingulate cortex responds differentially to expectancy violation and social rejection. Nat Neurosci 9:1007-1008.

Starzyk KB, Holden RR, Fabrigar LR, MacDonald TK (2006) The personal acquaintance measure: a tool for appraising one's acquaintance with any person. J Pers Soc Psychol 90:833-847.

Talairach J, Tournoux P (1988) Co-planar stereotaxic atlas of the human brain: an approach to medical cerebral imaging. New York: Thieme Medical Publisher.

van Winden F, Stallen M, Ridderinkhof R (2008) On the nature, modeling, and neural bases of social ties. In: Advances in health economics and health sciences research, Volume 20: neuroeconomics (Houser D, McCabe K, eds), pp 125-160. Bingley, UK: Emerald Group. 